\title{
Force Spectroscopy Imaging and Constriction Assays Reveal the Effects of Graphene Oxide on the Mechanical Properties of Alginate Microcapsules
}

\author{
María Virumbrales-Muñoz, Laura Paz-Artigas, Jesús Ciriza, Clara Alcaine, Albert Espona-Noguera, \\ Manuel Doblaré, Laura Sáenz del Burgo, Kaoutar Ziani, Jose Luis Pedraz, Luis Fernández,* \\ and Ignacio Ochoa*
}

Cite This: https://dx.doi.org/10.1021/acsbiomaterials.0c01382

Read Online

\section{ACCESS | Lلll Metrics \& More | 回 Article Recommendations | (s) Supporting Information}

ABSTRACT: Microencapsulation of cells in hydrogel-based porous matrices is an approach that has demonstrated great success in regenerative cell therapy. These microcapsules work by concealing the exogenous cells and materials in a robust biomaterial that prevents their recognition by the immune system. A vast number of formulations and additives are continuously being tested to optimize cell viability and mechanical properties of the hydrogel. Determining the effects of new microcapsule additives is a lengthy process that usually requires extensive in vitro and in vivo testing. In this paper, we developed a workflow using nanoindentation (i.e., indentation with a nanoprobe in an atomic force microscope) and a custom-built microfluidic constriction device to characterize the effect of graphene oxide

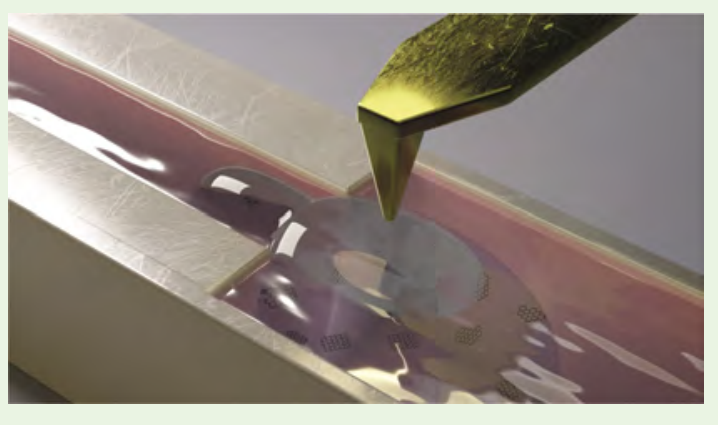
(GO) on three microcapsule formulations. With our workflow, we determined that GO modifies the microcapsule stiffness and surface properties in a formulation-dependent manner. Our results also suggest, for the first time, that GO alters the conformation of the microcapsule hydrogel and its interaction with subsequent coatings. Overall, our workflow can infer the effects of new additives on microcapsule surfaces. Thus, our workflow can contribute to diminishing the time required for the validation of new microcapsule formulations and accelerate their clinical translation.

KEYWORDS: graphene oxide, alginate, cell microencapsulation, force spectroscopy, stiffness, topography

\section{INTRODUCTION}

In the past few decades, regenerative cell therapy has demonstrated encouraging successes in the treatment of many diseases, using approaches such as cell encapsulation. ${ }^{1,2}$ Cell encapsulation aims to immobilize cells within a hydrogelbased porous micro- or macrosphere that allows nutrients to diffuse inside and therapeutic products (e.g., insulin and erythropoietin) to be secreted outside, while avoiding the immune system surveillance. ${ }^{3}$ The effectiveness of cell encapsulation relies on the successful concealing of exogenous cells and materials, which requires adequate mechanical resistance to prevent microcapsule rupture and the subsequent content release. ${ }^{4}$ Currently, the main challenges of microencapsulated cell therapy include the accumulation of dead cells inside the microcapsules and the optimization of the sustained product release from encapsulated cells to achieve long-lasting treatments. ${ }^{4,5}$ To overcome these challenges, classical microcapsule formulations have been supplemented with additives or coatings, thereby modifying microcapsule properties (i.e., hybrid microcapsules). ${ }^{6}$ Specifically, the combination of graphene oxide (GO) and microencapsulation technology has shown to improve the long-term alginate- encapsulated cell survival and sustainable therapeutic protein release, ${ }^{7}$ without triggering a foreign body response. ${ }^{4}$

$\mathrm{GO}$ is used in a wide range of biological applications (e.g., drug delivery, tissue engineering, and biosensing).$^{8-11}$ One of the reasons behind the popularity of GO as an additive is its excellent mechanical properties. ${ }^{12}$ Specifically, GO has been shown to improve the tensile strength of porous biomaterials without modifying their pore size or thickness, ${ }^{13}$ which is crucial for maintaining the diffusivity of nutrients into the capsules and keep encapsulated cells viable. ${ }^{14}$ Interestingly, low concentrations of GO have been reported to increase the viability of encapsulated cells. ${ }^{7}$ Although the incorporation of GO in new microcapsule formulations may be advantageous for cell viability, questions remain on the potential effects of $\mathrm{GO}$ on the alginate hydrogel structure and resulting

Received: September 17, 2020

Accepted: December 9, 2020 

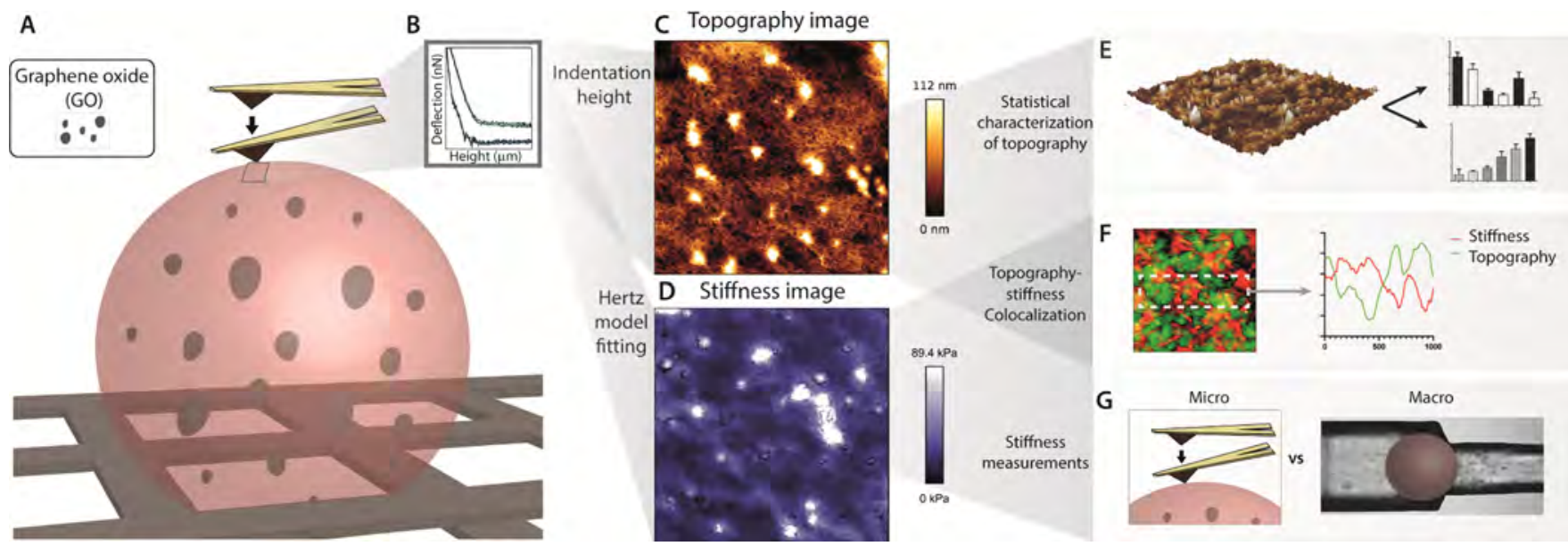

Figure 1. Schematic representation of the FSBI methodology. (A) Microcapsules were immobilized within a nylon mesh to acquire the (B) spectroscopy-based measurements via AFM. (C,D) Force spectroscopy-based grids were acquired for each microcapsule and processed to obtain both (C) a topography and (D) a stiffness map of the same microcapsule surface area. (E) Schematic representing the characterization of bidimensional statistical quantities for the topography images. (F) Overlays of topography and stiffness maps were generated to determine the colocalization degree of both parameters. $(G)$ Stiffness measurements obtained via FSBI were compared to macroscopic measurements via constriction assays with a custom-designed microfluidic system.

mechanical properties. The effects of new additives are of particular importance on the microcapsule surface, where the interactions between the host cells and the microcapsule material occur. As discussed previously, unstable outermost interfaces of microcapsules can have a profound impact on performance and biocompatibility. ${ }^{15}$ Therefore, to refine formulations prior to in vivo testing and advance regenerative cell therapy more quickly, it is critical to develop techniques to characterize the mechanical and surface properties of newly designed microcapsule formulations.

Previous studies have used infrared spectrophotometry by Fourier transform-attenuated total reflectance (ATR-FTIR) to characterize the effects of hybrid microcapsule compositions on surface polymer conformations. However, this technique provides an insight within a small depth of the microcapsule (100 ̊) and remains challenging to interpret. ${ }^{16}$ Scanning electron microscopy (SEM) is a valuable technique to characterize surfaces. However, most SEM sample preparation procedures modify the hydrogel structure, thereby providing inaccurate surface characterizations. ${ }^{17}$ Conversely, atomic force microscopy (AFM) is a powerful set of techniques for the characterization of surface properties of materials, which does not rely on sample modifications. ${ }^{18-20}$ Lately, a small number of studies have used AFM to characterize microcapsule surfaces under liquid physiological environments. ${ }^{14,21-23}$ The nanometric resolution of AFM can provide accurate data of the microcapsule surface topography along with local stiffness measurements at the surface level. ${ }^{24}$ In previous work, we used a popular and powerful AFM technique to acquire simultaneous topography and stiffness maps of the microcapsule called force spectroscopy-based imaging (FSBI). ${ }^{22,25-28}$ Variations of this technique have been used since before the 2000s by Hinterdorfer and others to produce quantitative nontopography images of antibody-antigen recognition forces, among other molecular interactions and physical properties. ${ }^{28-32}$ In our previous paper, we leveraged, for the first time, FSBI along with a stiffness-topography colocalization analysis to investigate the effects of microcapsule additives on the polymer conformation. This combination of techniques provided us with useful information on the surface properties of the microcapsules, but this alone is not enough to determine the microcapsule's overall stiffness. In addition, stiffness determination via nanoindentation is useful only for small strains, whereas hydrogel microcapsules are often subjected to large strains.

Microcapsules are subjected to high shear stresses and considerable strains, as they are forced to go through a rigid needle in the injection process. ${ }^{33}$ Excessive shear stress may result in microcapsule breaking and thereby releasing its contents, which could elicit an immune response. ${ }^{34}$ Although $\mathrm{AFM}$ is a sensitive technique for characterizing topography and surface stiffness, ${ }^{23}$ assessing global stiffness calls for a different approach, especially for the large strains exerted in the injection process. Shear stress application assays via micromanipulation, aspiration, or constriction have been used to investigate the overall stiffness of microcapsules. ${ }^{35-37}$ Experimental determination of stiffness for large deformations is not trivial because of the inelastic behaviors of the material and uneven compressions arising from the spherical geometries. ${ }^{33}$ However, a constriction test (i.e., forcing the capsules to pass through a narrow channel) can be used to approximate pressure/deformation relationships to compare different microcapsule compositions, ${ }^{38}$ as a complementary set of measurements to FSBI-based surface topography and stiffness.

Herein, we used two complementary methods for the characterization of hybrid microcapsule formulations. First, we used FSBI to analyze the effects of GO on surface features and mechanical properties of three different alginate-based microcapsules. In particular, we tested the effects of GO on alginate microcapsules (A), alginate microcapsules with a double polyL-lysine (PLL)-alginate coating (alginate-PLL-alginate, APA), and liquefied APA microcapsules (i.e., treated with sodium citrate to produce a softer capsule). ${ }^{39}$ Using FSBI, we characterized the topography of the microcapsule surface and the stiffness at the surface level. In parallel, we also developed a custom-designed constriction test to perform global stiffness measurements of the microcapsules. Finally, we established correlations between topography and surface stiffness to determine the effects of $\mathrm{GO}$ on the microcapsule surface and polymer conformation. Our results show that the effect of GO 

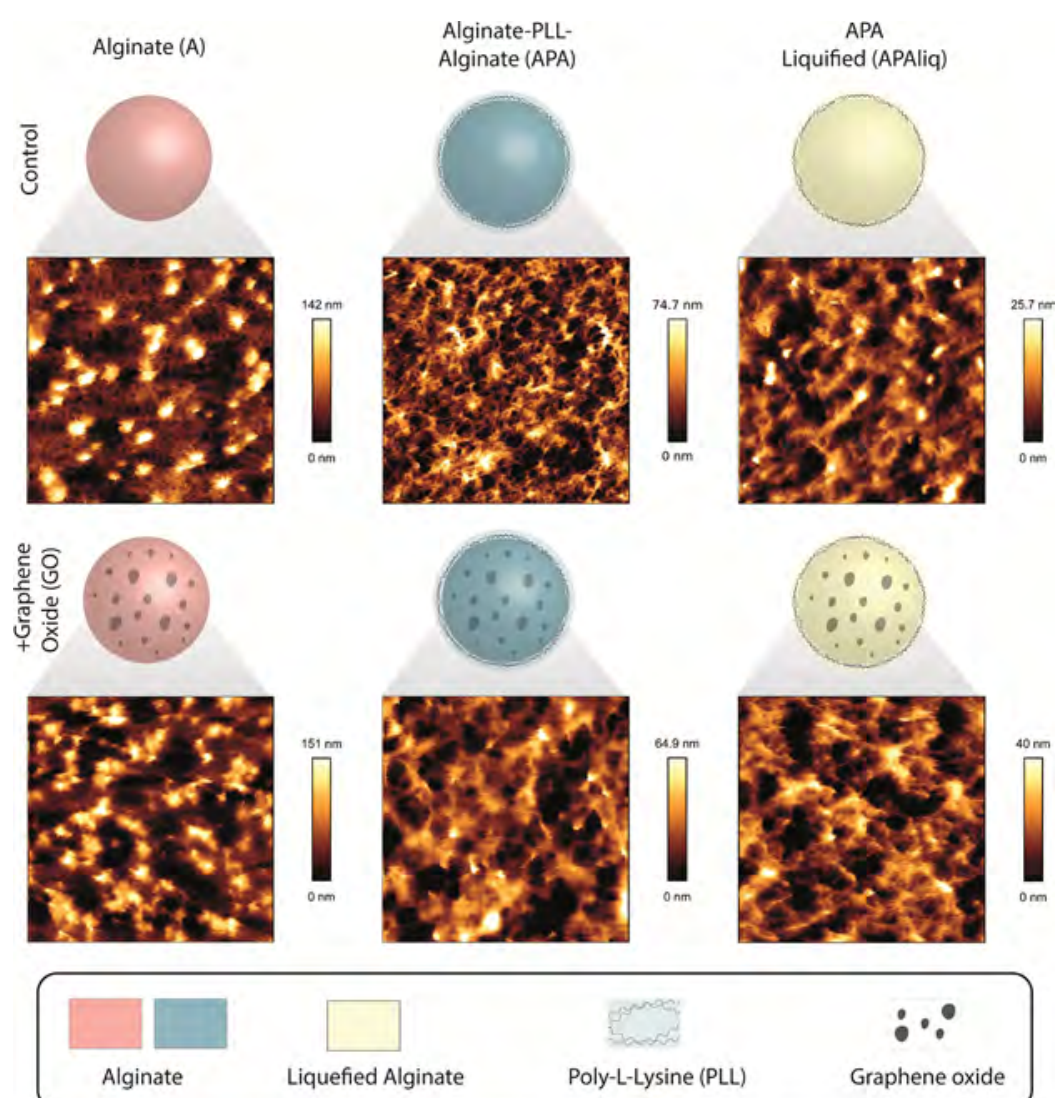

Figure 2. Schematic of the three different microcapsule formulations and their GO-supplemented counterparts with representative microcapsule topography images of each one acquired via FSBI. Alginate microcapsules are produced by extrusion of an alginate solution in an electrostatic atomization generator. These microcapsules are treated first with a poly-L-lysine coating and second with an alginate coating to produce APA microcapsules. APAliq microcapsules are obtained by incubating APA microcapsules in a sodium citrate solution. To obtain their GOsupplemented counterparts, the initial alginate solution is supplemented with $100 \mu \mathrm{g} / \mathrm{mL}$ GO. Topography images $\left(9 \mu \mathrm{m}^{2}\right)$ of each microcapsule were acquired to analyze topographical differences resulting from the different microcapsule compositions. Height scale bars are adjusted to optimize the visualization of topography details.

depends on the microcapsule formulation. Particularly, our results suggest that GO modifies the stiffness-topography distribution, thereby suggesting that GO alters the conformation of the alginate hydrogel, as well as coating binding to alginate. The workflow comprising these two techniques (FBSI and microcapsule constriction) can be used to assess the mechanical properties resulting from hybrid microcapsule formulations in vitro. We anticipate that the use of these two techniques, in combination, will help accelerate microcapsule formulations and, eventually, their use in clinical applications.

\section{RESULTS AND DISCUSSION}

This study was motivated by previous work from us and others, indicating that supplementing microcapsules with GO particles within a limited size $(1535.33+435.31 \mathrm{~nm})$ and concentration range $(25-50 \mu \mathrm{g} / \mathrm{mL})$ could be beneficial in keeping high cell viability of encapsulated cells and did not have a negative impact on the viability of implanted microcapsules. $4,7,14$

We characterized the microcapsule surface features by FSBI to analyze the effects of GO on the surface of alginate-based hybrid microcapsules. To this end, we used nanoindentation measurements, performed at $37^{\circ} \mathrm{C}$ in liquid (DPBS), to mimic physiological conditions after microcapsule implantation. The microcapsules were immobilized in a nylon mesh, thereby avoiding sample preparation techniques that may damage the original structure and properties of the microcapsules (Figure
1A). Each force spectroscopy curve was acquired within a grid and corresponded to a pixel (Figure 1B), thereby generating map images of the microcapsule surface (Figure $1 \mathrm{C}$ ). On the one hand, topography map images (gold) were obtained by depicting the height values calculated from the force spectroscopy measurements. On the other hand, the same force spectroscopy curves were fitted to a Hertz model, yielding Young's modulus values that rendered stiffness map images (blue). These FSBI maps were then analyzed to characterize microcapsule topographical (Figure 1E) and mechanical properties (Figure 1F,G).

Accurate Hertz model fittings to calculate Young's moduli require specific types of tip conformations (e.g., spherical, quadratic pyramid, and conical). ${ }^{40,41}$ Notably, our choice of cantilever shape was quadratic pyramid, as opposed to the most popular one for hydrogel measurements but more costly colloidal/spherical tips. In our case, pyramid-shaped tips were chosen because of their potentially higher stability in measuring spherical samples, as well as their much lower cost, which was more convenient for our large sample size. Importantly, our study illustrates that a more affordable tip shape can be successfully used to acquire stable topographies and curves that fit Hertz model variations successfully.

To determine the effect of GO in hybrid microcapsules, we studied three microcapsule formulations: basic alginate (A) microcapsules, classical alginate-poly-L-lysine-alginate micro- 
A Roughness parameters

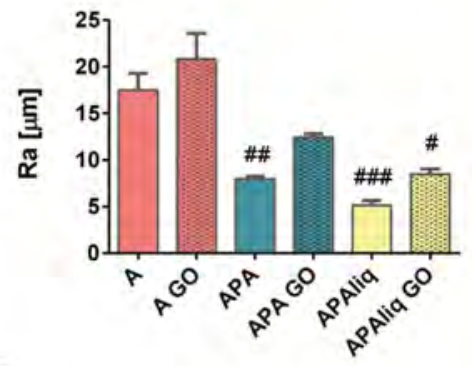

B

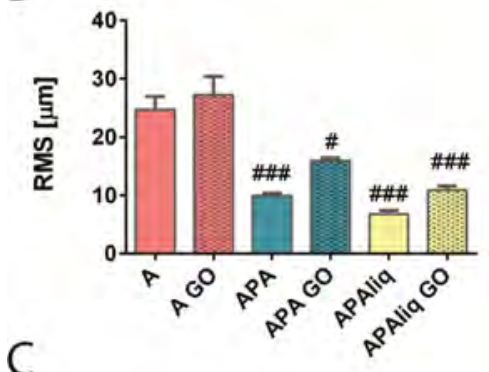

C

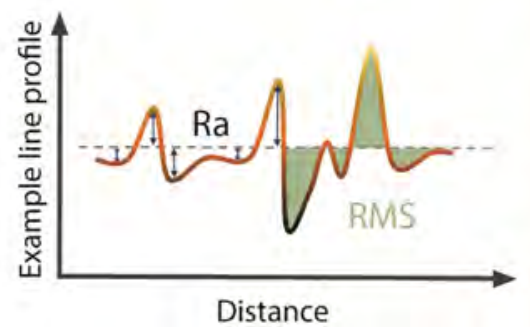

D Image height

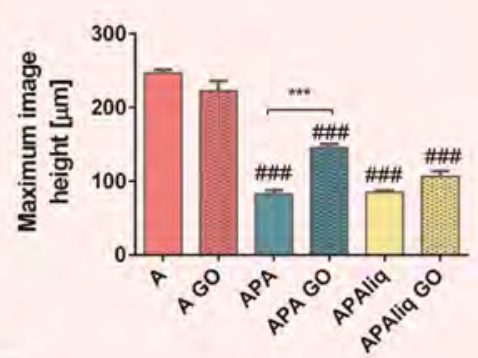

E
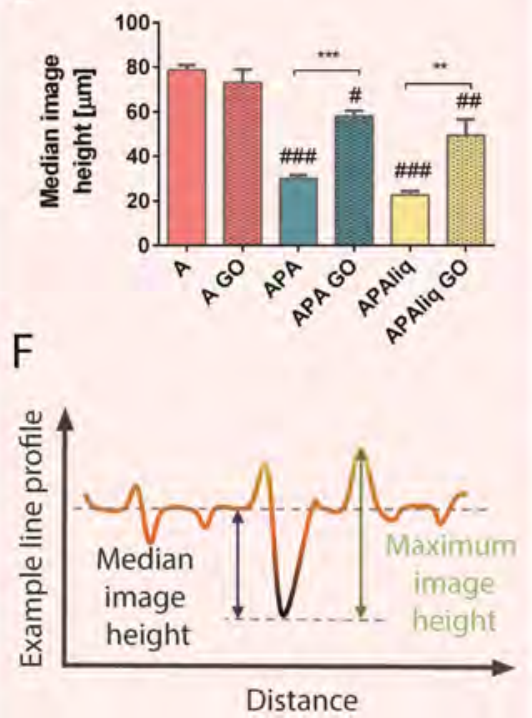

G Height distribution ratio

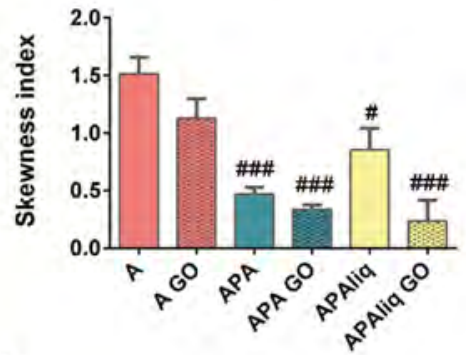

$\mathrm{H}$
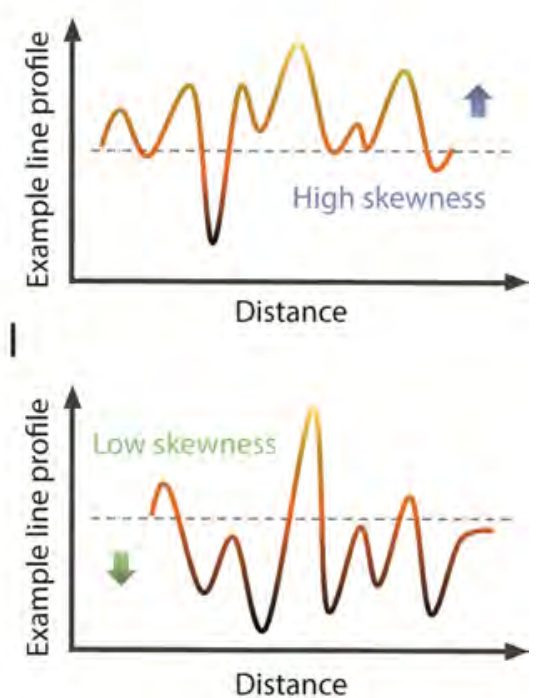

Figure 3. Topographical study of microcapsule surfaces. Standard topography parameters calculated from topography maps $\left(9 \mu \mathrm{m}^{2}\right)$ of microcapsules. (A,B) Graphs showing roughness parameters: (A) Ra and (B) RMS, (C) Schematic representation of the significance of Ra and RMS on an example line profile. (D,E) Graphs showing image height parameters. (D) Maximum image height, (E) median image height, and (F) schematic representation of maximum and median image height on an example line profile. (G) Graph showing skewness, a measurement of height distribution in an image. (H,I) Schematic representations of (H) high and (I) low skewness on an example line profile. $N \leq 5$ samples per microcapsule composition. Graphs show mean \pm SEM. Two-way ANOVA showed independence between GO effects and microcapsule formulation effects on roughness and height distribution, while it showed interdependence of these effects on height parameters. Sidak's multiple comparisons tests were used to compare GO versus control capsules and each formulation versus A. * = significance of GO versus control capsules. $\#$ = significance compared to A microcapsules. $* p<0.05 ; * p<0.01 ; * * p<0.001$.

capsules (A-PLL-A or APA), and APA microcapsules liquified via sodium citrate treatment (APAliq). For each microcapsule composition type, $100 \mu \mathrm{m}^{2}$ topography maps (Figure S1) and $9 \mu \mathrm{m}^{2}$ topography maps (Figures 2 and S2) were collected. Larger images inform the homogeneity of microcapsule topography, whereas smaller view images provide enough resolution to capture topography features. Therefore, the analysis was performed with the $9 \mu \mathrm{m}^{2}$ topography maps alone. Qualitatively, topographic maps of control and GOsupplemented capsules did not seem different from each other. Conversely, topographic maps of coated (i.e., APA and APAliq) microcapsules appear different from naked alginate (A) microcapsules. More specifically, alginate (A) capsules presented a higher prevalence of thick peaks and extensive valleys. The appearance of the surface was consistent with previous reports of the topography of the alginate cross-linked network structure. ${ }^{42,43}$ Conversely, in APA and APAliq microcapsules, the surface presented a porous structure, consistent with features expected from the alginate-PLL coating. $^{21,22}$

To perform a more exhaustive analysis of the effects of GO on microcapsule topography, bidimensional statistical quantities were calculated from all FSBI topography maps (Figure 3): $\mathrm{Ra}$, RMS, maximum image height (MaxIH), median image height (MedIH), and skewness. ${ }^{44,45}$

We first investigated surface roughness by calculating $\mathrm{Ra}$ (Figure 3A) and RMS (Figure 3B) parameters. Both parameters calculate deviations of the surface from a flat plane. $\mathrm{Ra}$ calculates this deviation as the distance that the surface of interest deviates from a flat plane, whereas RMS calculates the deviations as the area enclosed by these two components (Figure 3C). ${ }^{46}$ A two-way analysis of variance showed that Ra and RMS are independently affected by both microcapsule formulation and GO. Hence, roughness is significantly influenced by microcapsule composition (Ra: $F(2,34)=33.39$ and $P<0.001$; RMS: $F(2,34)=48.99$ and $P<$ 
A Topography
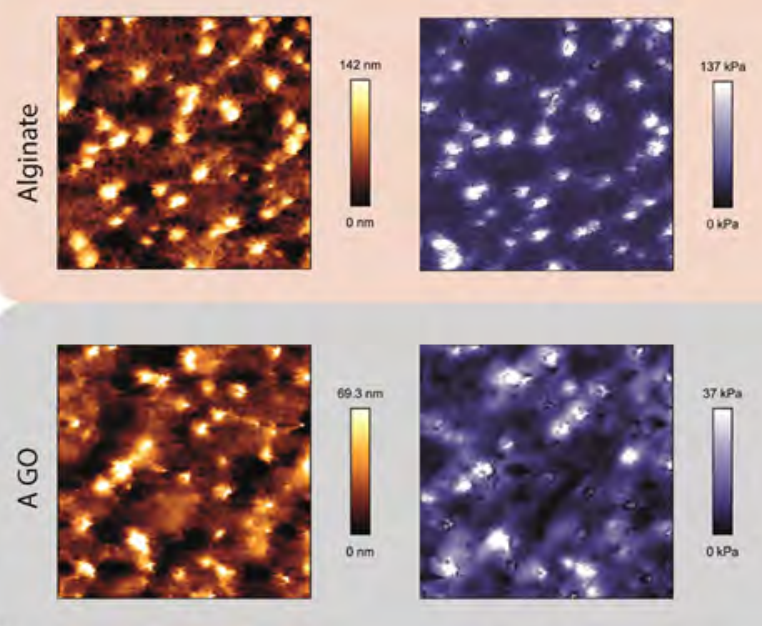

$\mathrm{E}$

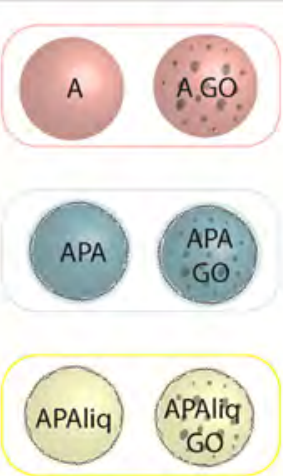

Stiffness

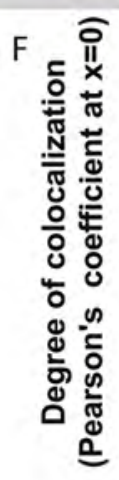

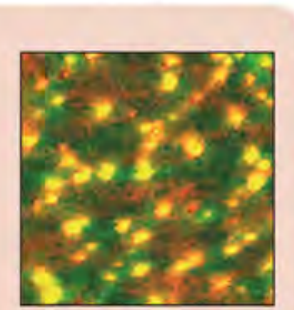

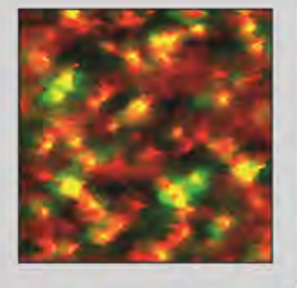

Overlay

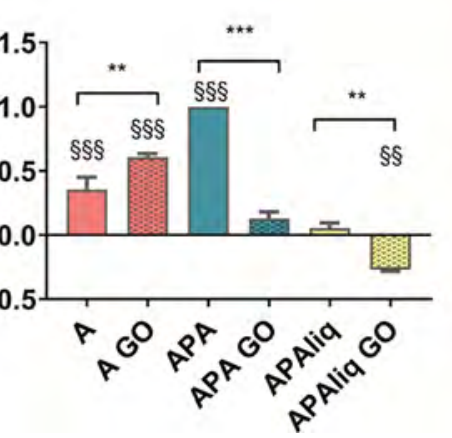

B
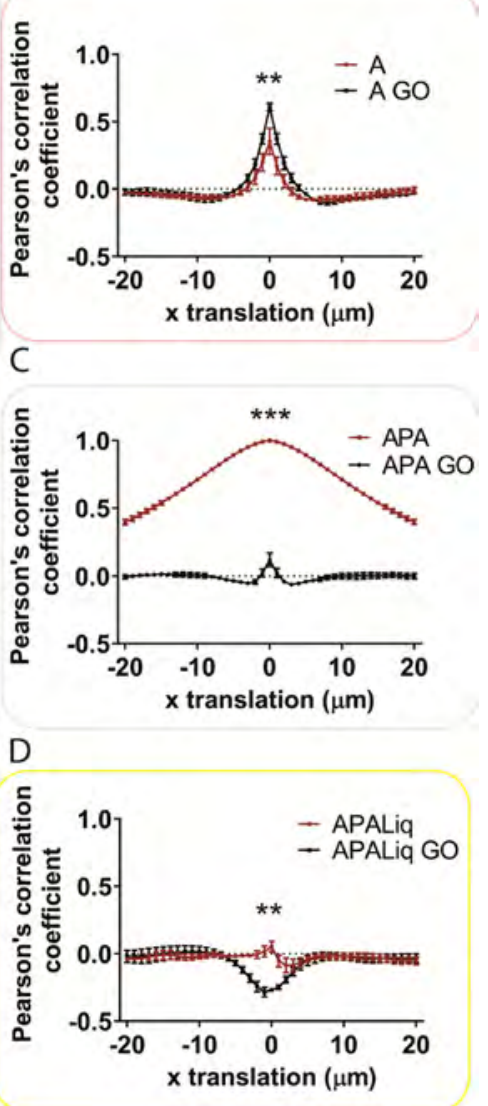

Figure 4. Representative microcapsule stiffness maps obtained by FSBI. (A) Topography and Young's modulus distribution maps $\left(9 \mu \mathrm{m}^{2}\right)$ of each microcapsule were acquired to establish topography-stiffness colocalization differences between microcapsule compositions. Sample images for alginate and A GO capsules are depicted, including topography and stiffness maps and overlays (green = stiffness, red = topography, and yellow = colocalizing structures). (B-D) Pearson's correlation coefficients were calculated using the van Steensel method for all capsules. Each GOsupplemented microcapsule formulation was compared to its control counterpart. (B) Pearson's correlation coefficient comparisons of A vs A GO, (C) APA vs APA GO, and (D) APAliq versus APAliq GO microcapsules. (E) Schematic representation of all microcapsule formulations with their assigned color code. (F) Degree of colocalization presented as the Pearson coefficient at $x=0$ from graphs (B-D) for comparison among formulations. Significant differences between each pair of compared formulations were calculated via one-way ANOVA and post hoc Tukey test. * $=$ significance of GO vs control microcapsules. $\S=$ significance compared to $0 . * * p<0.01 ; * * p<0.001$.

0.001) (a more detailed explanation of the two-way ANOVA results can be found in Figure S3). In particular, coated microcapsules present smaller values of Ra and RMS than A microcapsules. As previously suggested, the coating seems to have a smoothing effect on the microcapsule surface. ${ }^{22}$ Meanwhile, GO increases surface roughness in supplemented microcapsules (Ra: $F(1,34)=8.564$ and $P=0.006$; RMS: $F(1,34)=8.067$ and $P=0.008)$.

Next, we assessed the height distributions of our topography maps by calculating MaxIH and MedIH. MaxIH (Figure 3D), or surface amplitude, is the absolute distance between the lowest and the highest point of the surface map, whereas MedIH (Figure 3E) corresponds to the most prevalent height value throughout the surface. Therefore, MedIH can be understood as the surface baseline from which peaks and valleys emerge (Figure 3F). Consistent with roughness results, both MaxIH and MedIH are affected by microcapsule formulation (MaxIH: $F(2,34)=156.7$ and $P<0.001$; MedIH: $F(2,34)=53.22$ and $P<0.001)$. We found that coated microcapsules have lower height values than $A$ microcapsules, consistent with a flattening effect of the coating.
However, the addition of GO produces the opposite effect, increasing MedIH and MaxIH of the microcapsule surfaces (MaxIH: $F(1,34)=8.211$ and $P=0.007$; MedIH: $F(1,34)=$ 23.88 and $P<0.001)$. Interestingly, there is an interaction between the microcapsule formulation and the addition of GO in both height parameters (MaxIH: $F(2,34)=13.01$ and $P<$ 0.001; MedIH: $F(2,34)=10.85$ and $P<0.001)$. Overall, we observed that GO increases height preferentially in coated capsules, with no effect on the already irregular surface of naked A capsules. Notably, MaxIH in APAliq microcapsules was unaffected by the addition of GO, which could be explained by the citrate treatment on the surface of the microcapsule, resulting in flatter surfaces in both control and GO-supplemented capsules.

The last bidimensional statistical quantity we analyzed was skewness (Figure 3G). Skewness is an indicator of the surface's irregularity and represents the symmetry (or lack thereof) of the height distribution compared to half of the MaxIH (Figure $3 \mathrm{H}, \mathrm{I})$. It is important to note that differences in skewness are calculated according to the height of each image. We found that this parameter was strongly dependent on microcapsule 

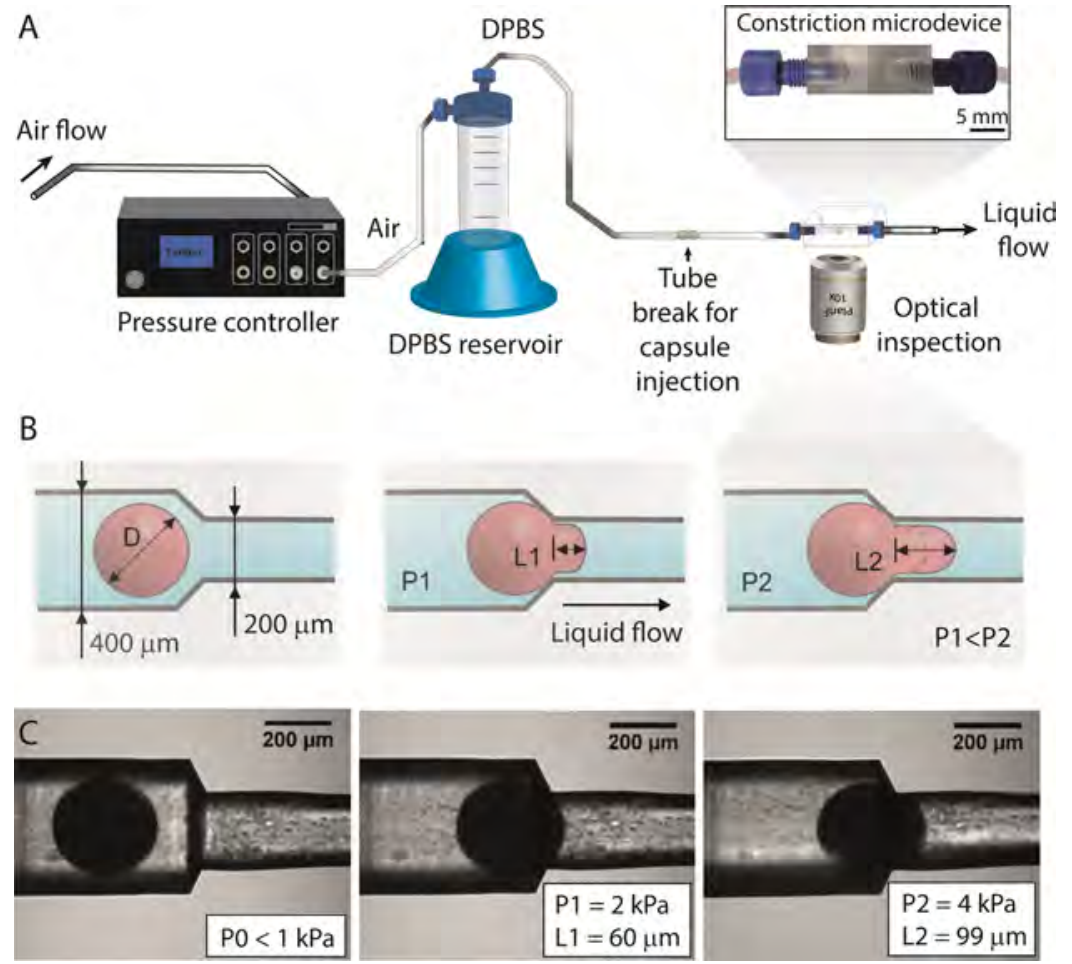

Figure 5. Microcapsule constriction assay. (A) Graphic depiction of the constriction system set up. (B) Scheme of the constriction assay process and parameters needed for calculating our elastic constant: initial diameter (indicated as $D$ ) of every microcapsule and penetration length (indicated as $L$ ) are optically measured. At the same time, the pressure controller sets pressure (marked as $P$ ) at the inlet. (C) Microscopy images (Leica) of a microcapsule constriction assay. Hematoxylin stain of the microcapsule for visualization purposes.

formulation $(F(2,34)=28.07$ and $P<0.001)$. Notably, A microcapsules showed the highest skewness values, suggesting that naked surfaces were mostly asymmetrical (i.e., presenting more peaks and shallow valleys), which is consistent with our initial observations (Figure 2). Conversely, coated microcapsules presented valleys with a smaller diameter and more discrete peaks. Overall, although we did not see differences between control and hybrid microcapsules, a two-way analysis revealed that the addition of GO resulted in a more skewed down surface $(F(1,34)=12.39$ and $P=0.001)$ regardless of microcapsule formulation.

In previous work, we investigated the colocalization of simultaneously acquired topography and stiffness maps to study alginate chain-coating interactions on the microcapsules' surface. $^{22}$ Reportedly, GO has excellent mechanical properties (i.e., presents a high Young's modulus), which could disrupt the typical conformation of the softer alginate and PLL components of the microcapsules. ${ }^{47}$ In this study, topography-stiffness colocalizations could inform the location of GO within the microcapsule and its effect in the alginate conformation on the surface. Hence, for each topographical map collected, we also obtained its stiffness counterpart via Hertz model fitting of each force spectroscopy curve (Figures S4 and S5 for 100 and $9 \mu \mathrm{m}^{2}$ maps, respectively). Mean values of these stiffness maps were calculated (Figure 6) to compare surface stiffness of each microcapsule formulation.

With these data, we first studied whether the GO added to the microcapsules was located close enough to the surface to detect it via FSBI. To do so, we determined the Young's modulus for GO in our system by performing the FSBI technique on GO from the suspension used to supplement our microcapsule formulations (Figure S6), under the same conditions as used for microcapsules. If GO is located close to the microcapsule surface, higher stiffness values would be measured via FSBI than in the control formulations. Although the low cantilever stiffness may not be ideal to determine the absolute stiffness values of $\mathrm{GO}$, it provides an accurate control to determine the presence of $\mathrm{GO}$ in microcapsules. The stiffness values of GO $\left(1.19 \pm 0.812 \times 10^{4} \mathrm{kPa}\right)$ were over 40 fold above the maximum values of APA GO microcapsules (Figures S3 and S4), which presented the highest stiffness in our study. Furthermore, GO-supplemented formulations were less stiff than their control counterparts. Hence, we concluded that GO was not within a detectable range of the surface for our technique.

Then, we investigated the effect of GO on the topographystiffness correlation of our microcapsule formulations. Thus, we overlaid topography (gold) and stiffness (blue) maps (Figure 4A; sample stiffness maps in Figures S3 and S4) from the same region. Using the Van Steensel method, we calculated Pearson's correlation coefficient for each pair of microcapsule formulations (i.e., control and GO-supplemented) (Figure $4 \mathrm{~B}-\mathrm{F}$ ). Control formulations presented significant stiffnesstopography colocalization (i.e., the peaks in topography were stiffer than the rest of the surface). However, we observed that the presence of GO significantly changed the degree of colocalization of the control formulations $(F(2,44)=85.77$ and $P<0.001)$. Interestingly, the overall effect and direction of this disruption depended on the microcapsule formulation $(F(2,44)=67.62 ; P<0.001)$. Particularly, in the alginate (A) microcapsules, the addition of GO resulted in a higher degree of colocalization between topography and stiffness. In other words, the topography peaks corresponded more precisely to stiffer regions of the GO-supplemented microcapsule surfaces. 
Conversely, in APA and APAliq formulations, the addition of GO significantly decreased the microcapsules' stiffnesstopography colocalization. Surprisingly, the colocalization degree in APAliq was close to zero, indicating no topography-stiffness correlation, whereas in APAliq GO, the colocalization values were negative (i.e., the topography peaks and stiffness peaks did not coincide with each other).

These results indicate that the presence of GO disrupts the polyanionic alginate matrix significantly on the surface, even though the FSBI does not directly detect the GO. Notably, this decrease in degree of colocalization appears in the two coated formulations (i.e., APA and APAliq). This result could indicate that microcapsules with subsequent coatings or treatments presented a looser attachment between the coatings and the polyanionic matrix.

Finally, we analyzed the effect of GO on microcapsule stiffness. However, FSBI is based on nanoindentation, and thus, it provides only surface measurements and for small deformations. Therefore, we assessed global average stiffness via a custom-made constriction assay (schematic in Figure 5A). In this assay, microcapsules in a DPBS solution were forced to pass through a narrow channel. This assay mimics the shear stress inflicted on microcapsules during the implantation process, and stiffness is calculated as a pressure-deformation ratio (Figure 5B,C). The average stiffness results from this constriction assay were compared to the median value of stiffness maps obtained via nanoindentation (Figure 6).
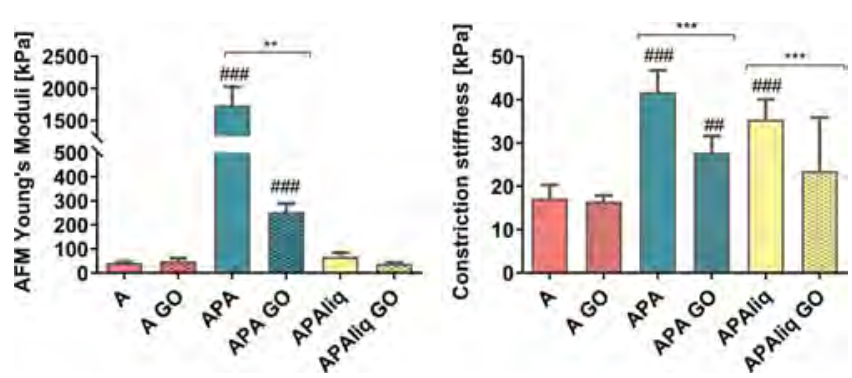

Figure 6. Results of microcapsule stiffness analysis. (Left) Young's modulus values obtained from stiffness maps via nanoindentation (force spectroscopy) of the microcapsule surface. $n \leq 5$ per microcapsule composition group, from two different batches of microcapsules. (Right) Whole microcapsule constriction stiffness as a pressure-deformation ratio obtained from constriction assay. $n=$ at least 10 per formulation. Graphs represent mean values \pm SEM. The unpaired $t$-test was used to compare GO vs control capsules and each formulation vs $\mathrm{A} . *$ s significance of $\mathrm{GO}$ versus control capsules. \# = significance compared to A microcapsules. ${ }^{*} p<0.05$; $* * p<0.01$; $* * * p<0.001$.

The constriction of microcapsules through a smaller tube is a complex mechanical problem. Microcapsule constriction incurs in a wide range of strains and stresses, generating substantial deformations throughout the microcapsule geometry and with a high variation throughout its geometry. Although the elastic modulus is commonly used in mechanical problems where small strains are applied (e.g., nanoindentation), speaking of pressure-deformation ratios in terms of elastic modulus is inaccurate, because a great variety of tangent moduli, which could be considered as the strain-dependent Young's modulus counterpart, are simultaneously produced pointwise. Only a comprehensive simulation of the whole process (e.g., via finite elements and parameter fitting of the material constants for the microcapsules) would accurately define the microcapsule stiffness, without considering the inhomogeneity induced by GO distribution. However, because our main goal is to compare the relative stiffness between different types of microcapsules, the proposed pressure-deformation ratio, which averages the microcapsule stiffness through the first state of the constriction process, is sufficient for our purpose. Thus, our comparisons between both stiffness measurements (Figure 6) will not rely on comparing absolute values but tendencies among the different formulations.

Our first observation in comparing the stiffness of the different formulations was the high stiffness of APA capsules via FSBI as compared to all other formulations, which was not observed in the constriction measurements. This difference highlights the difference between both techniques. In other words, although FBSI is only measuring surface stiffness, the constriction assay produces a more macroscopic measurement of stiffness and for larger strains. Despite these striking differences, both techniques (i.e., FSBI and constriction) revealed a decrease in stiffness in GO-supplemented formulations (FSBI: $F(1,59)=20.34$ and $P<0.001$; constriction: $F(1,65)=32.60$ and $P<0.001)$. Furthermore, the effect of GO strongly depends on the microcapsule formulation (FSBI: $F(2,59)=32.16$ and $P<0.001$; constriction: $F(2,65)=46.78$ and $P<0.001)$.

Particularly, the stiffness of the APA GO formulation was significantly decreased compared to that of APA, although it remained significantly stiffer than the other formulations. This result illustrates the high local stiffness of the classical APA design on the surface and suggests a significant impact of GO in the surface stiffness of this formulation. Although the impact of GO in stiffness is clear in both sets of measurements, it is in the APA formulation where the impact of GO was more prominent. This result is consistent with the hypothesis that both the bulk hydrogel and the coatings are impacted by the addition of GO. Interestingly, the decrease in stiffness in APAliq GO as compared to its control counterpart was not detectable via FBSI, which suggests that the main effect of GO in decreasing the stiffness of APAliq formulations occurs in the bulk of the alginate hydrogel, rather than on the surface. Also, most likely, its effect is more pronounced for higher local deformations of the polymer.

For this study, we chose to keep our cantilever measurements consistent by performing all of our FSBI measurements with the same type of cantilever. A caveat for using only one type of cantilever for the FSBI measurements is found in the stark differences between APA/APA GO formulation stiffnesses as compared to all other formulations. It is known that cantilevers perform best for samples in a specific range of stiffness; therefore, the top stiffness values may not be accurate measurements. In this study, we aimed to compare the different formulations to one another. Future studies could provide an accurate measurement of stiffness for these microcapsule formulations or the GO particles themselves using a stiffer cantilever to perform the FSBI.

Overall, in this paper, we determined that GO induced changes in all microcapsule formulations (summary in Table 1 ). The effect of GO on naked alginate capsules (A) was limited to a higher degree of colocalization between topography and stiffness, indicating that peaks were stiffer in the A GO formulation (dark blue color, Figure 7, left). Conversely, in both APA and APAliq formulations, there were significant changes in topography, namely, the increases in MaxIH (in 
Table 1. Summary of the Effects of GO on A, APA, and APAliq Microcapsule Formulations. “_” = No Effect

\begin{tabular}{|c|c|c|c|}
\hline & A vs A-GO & $\begin{array}{l}\text { APA vs } \\
\text { APA-GO }\end{array}$ & $\begin{array}{l}\text { APAliq vs } \\
\text { APAliq-GO }\end{array}$ \\
\hline $\begin{array}{l}\operatorname{maximum} \text { height } \\
(\text { MaxIH) }\end{array}$ & - & increase & - \\
\hline $\begin{array}{l}\text { median height } \\
(\text { MedIH })\end{array}$ & - & increase & increase \\
\hline roughness & - & increase & increase \\
\hline skewness & - & - & - \\
\hline $\begin{array}{l}\text { stiffness-topography } \\
\text { colocalization }\end{array}$ & $\begin{array}{l}\text { increase (peaks } \\
\text { are stiffer) }\end{array}$ & $\begin{array}{l}\text { decrease } \\
\text { (peaks are } \\
\text { softer) }\end{array}$ & $\begin{array}{l}\text { decrease } \\
\text { (peaks are } \\
\text { softer) }\end{array}$ \\
\hline surface stiffness & - & decrease & - \\
\hline overall stiffness & - & decrease & decrease \\
\hline
\end{tabular}

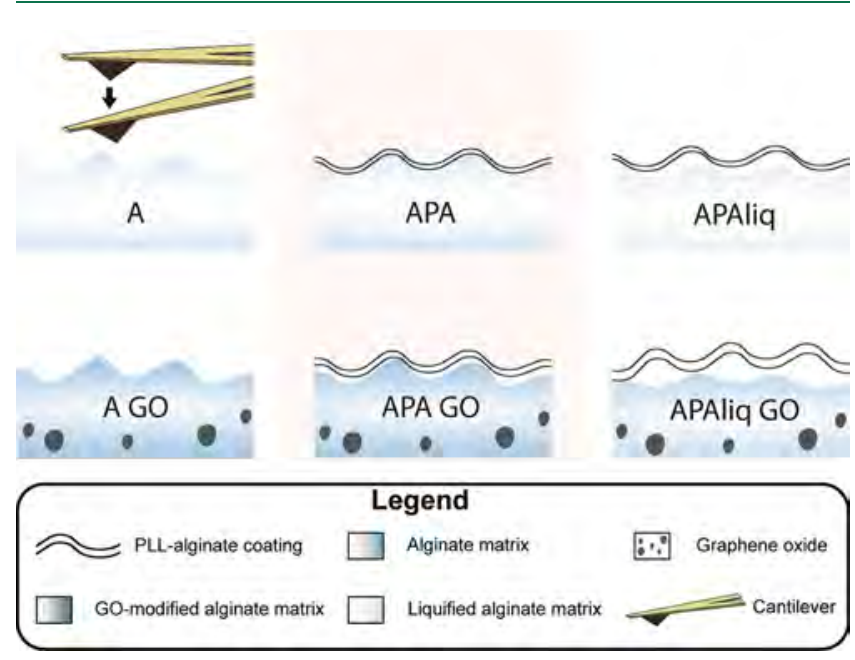

Figure 7. Proposed model of the surfaces of microcapsules before and after GO supplementation for alginate alone (A), APA, and APAliq microcapsules. A (alginate alone) microcapsules were less affected by the addition of GO, whereas APA formulations presented higher topographic features and weaker bulk hydrogels. Finally, APAliq microcapsules presented higher topographic features and a detachment of the PLL coating from the liquified alginate matrix.

APA alone) and MedIH (in both APA and APAliq) (Figure 7, center and right). The increase in MedIH is also consistent with the increase in surface roughness. Interestingly, the skewness (i.e., height distributions in the $z$ direction) of these formulations did not change as a result of the addition of GO. These two formulations also experienced a lower degree of colocalization between topography and stiffness, indicating that peaks were softer on both GO-supplemented formulations than in their control counterparts. This effect may be explained by a detachment of the PLL-alginate coating from the bulk alginate hydrogel. As we described in the previous section, GO had no effect on the stiffness of A capsules. Conversely, GO induced a weakening effect in the overall capsule of APA and APAliq. FSBI only detected a decrease in stiffness in APA GO versus APA microcapsules, suggesting that this weakening effect translated only to the surface of this formulation. Furthermore, our results indicate that subsequent modifications magnify the impact of GO on the surface topography and stiffness.

The impact of GO is consistent with our previous hypothesis, indicating that GO may be disrupting the typical alginate network structure. An explanation of the disruption of the alginate matrix could reside on the negative surface charge exhibited by $\mathrm{GO}^{48}$ Alginate microcapsules form a gel as a consequence of the ionic cross-linking of alginate chains and dicationic calcium. ${ }^{49}$ However, this electric charge equilibrium may be disturbed by GO, thereby changing the nature of the alginate polymer. Interestingly, the effect of GO seems to be more detectable in formulations with subsequent modifications or coatings. Next, we observed that the differences in topography were not significant in naked formulations, while they were significant in both APA and APAliq formulations. This increase in disorder with subsequent coatings is consistent with observations reported in our previous work. ${ }^{22}$

The effect of the liquefaction treatment on APAliq surfaces remains poorly described, and therefore, an in-depth interpretation of the effects of $\mathrm{GO}$ in this formulation remains challenging. However, our results show that this treatment generates profound differences in the alginate-PLL conformation. Although further investigation (e.g., z-potential measurements and ATR-FTIR) may be needed to elucidate the chemical modifications behind the topography and stiffness changes that we observed after the supplementation with GO, careful consideration of the adequacy of new formulations for each application is warranted.

Overall, we determined that GO has a detrimental effect on microcapsule stiffness. As for the significance of our findings, more compliant microcapsules would indicate a higher flexibility, and therefore, more highly adaptable microcapsules pass through a narrow needle during the implantation process. On the other hand, it is considered that a less robust microcapsule may release microcapsule contents and elicit an immune response. Therefore, care must be taken when subjecting GO-supplemented microcapsules to high shear stresses. In future studies, rupture pressures of microcapsules could be assessed to determine the impact of GO on this parameter. Another interesting point would be the effect of stiffer matrices on encapsulated cells. Matrix stiffness is known to impact cell function, ${ }^{50,51}$ while cell viability actually increased in GO-supplemented capsules, ${ }^{4}$ and the effects of stiffness on therapeutic product efficacy and secretion efficiency would be an interesting topic for future studies.

An increase in peak height or roughness in microcapsule surfaces has been discussed less in the literature. Such surface modifications are potentially disadvantageous for microcapsules for two reasons: (1) a larger surface of contact for microcapsules translates into more area for potential cellmicrocapsule interactions and therefore a higher potential for graft rejection and (2) there is evidence that an increase in roughness in biocompatible materials modifies cell adhesion and behavior. Further studies will need to be performed to determine whether the differences we detected using our workflow translate into significant disadvantages in vivo.

\section{CONCLUSIONS}

Microencapsulation is a promising approach to conceal cells from the host's immune system in cell-based therapy. However, a few hurdles remain for the full implantation of this technology in the clinic. Namely, the stability of the microcapsules to high shear stresses and guaranteeing a high cell variability are areas of current interest in the field of microencapsulation. The use of hybrid microcapsules has recently demonstrated to be successful in fulfilling these needs. Unfortunately, the success of the formulation is often found only after in vivo implantation, a costly and time-consuming process. 
In particular, the addition of GO to new microcapsule formulations has demonstrated potential in increasing cell viability without eliciting a foreign body response in vivo. Our results confirm changes in the mechanical properties of microcapsules as a result of including GO in microcapsule formulations. Importantly, although GO had an impact on the stiffness of microcapsules, it did not result in less stable formulations. Rather, once basic stability of microcapsules is achieved, GO-supplemented microcapsules may be more compliant to deformation and resilient to injection-related shear stress.

In this article, we used a workflow comprising FSBI and a constriction assay to uncover potential effects of GO on the microcapsule surfaces. Interestingly, we determined via FSBI that although GO is not located close to the microcapsule surface, it has an effect on the conformation of the alginate network, which becomes more apparent with subsequent coatings and surface modifications. Along with FSBI, our custom-made constriction assay could help in determining optimal microcapsule formulations to resist high stresses through the implantation process.

The use of this workflow to characterize the changes generated on the microcapsule surface as a result of changes in formulation could significantly shorten the time needed to validate each new formulation, along with costs, before testing them in vivo. Overall, we anticipate that our workflow will be used in the future as a tool to aid rational microcapsule design and help accelerate microencapsulation research and its clinical translation.

\section{MATERIALS AND METHODS}

4.1. Microcapsule Preparation. Microcapsules were generated following an adapted protocol to that of Lim and Sun. ${ }^{14,32}$ Briefly, procedures were carried out at room temperature under aseptic conditions. Mannitol (Sigma-Aldrich, M4125, Madrid, Spain) was dissolved at $1 \%$ in distilled water. Unless specified otherwise, subsequent solutions were prepared in this $1 \%$ mannitol aqueous solution. Ultrapure low viscosity high Guluronate, UPLVG, alginate (SKU: 4200001, NovaMatrix, FMC Biopolymer, Norway) was resuspended at final concentrations of alginate of $1.87,1.5$, and $0.1 \%$. Solutions were sterilized with a $0.22 \mu \mathrm{m}$ syringe filter (Millipore, MA, USA). Alginate solutions (1.5\%) were extruded in the electrostatic atomization generator VARV1 (Nisco, Switzerland) with a peristaltic pump (flow rate: $5.9 \mathrm{~mL} \cdot \mathrm{h}^{-1}$ ). The resulting microcapsules (A, or alginate alone) were collected in $55 \mathrm{mM} \mathrm{CaCl}_{2}$ (Sigma-Aldrich, Madrid, Spain) and incubated for $15 \mathrm{~min}$ in agitation to allow for complete gelation.

A + GO: To produce A capsules with GO, a $1.87 \%$ alginate solution with GO was used instead of the $1.5 \%$ alginate alone solution. To generate this solution, GO (Graphenea, Spain) was suspended in Milli-Q water at $250 \mu \mathrm{g} / \mathrm{mL}$ and sonicated for $1 \mathrm{~h}$ at high intensity. GO suspension was diluted to $1: 5(\mathrm{~V} / \mathrm{V})$ with $1.87 \%$ alginate solution to produce a $1.5 \%$ alginate solution and $100 \mu \mathrm{g} / \mathrm{mL}$ GO. Both "A" and "A GO" were washed and coated according to the desired microsphere formulation:

APA is a classical alginate-PLL-Alginate ( PLL = poly-L-lysine hydrobromide $M_{\mathrm{w}} 15-30 \mathrm{kDa}$ ) (Sigma-Aldrich, St. Louis, MO, USA) design. To produce these microcapsules, A microcapsules were incubated in a $0.05 \%(\mathrm{w} / \mathrm{v})$ PLL solution with agitation for $5 \mathrm{~min}$, followed by a second $0.1 \%$ alginate coating for another $5 \mathrm{~min}$.

Finally, to generate liquefied APA microcapsules (APAliq), APAcollected microcapsules were incubated in a $1.5 \%$ sodium citrate in $1 \%$ mannitol solution with agitation for $10 \mathrm{~min}$, as previously described. ${ }^{39}$ All microcapsules were finally washed twice with DPBS with calcium and magnesium (Sigma, D8662) and maintained in DPBS with $\mathrm{Ca}$ and $\mathrm{Mg}$. Resulting microcapsule preparations were A,
A GO, APA, APA GO, APAliq, and APAliq GO. Microcapsule morphology and diameter were examined under an inverted optical microscope (Nikon TSM, Japan).

4.2. AFM Setup and Measurements. Topographic and mechanical characterization of the microcapsules was performed by AFM (JKP Instruments). A Nanowizard 3 AFM module was used. The AFM system was mounted over an optical inverted microscope (Nikon-Eclipse).

4.2.1. Tip Calibration. AFM measurements were performed using a PNP-TR probe (NanoAndMore, Switzerland), tip A. This probe and tip were selected because of the low force constant $\left(0.32 \mathrm{~N} \cdot \mathrm{m}^{-1}\right)$, adequate soft materials, and its quadratic pyramidal shape $(3.5 \mu \mathrm{m}$ high, $<10 \mathrm{~nm}$ diameter) that allows a high resolution. The experimental spring constant of each tip used was empirically determined after a force spectroscopy measurement on a glass coverslip by the thermal noise method before every set of measurements. ${ }^{53}$

4.2.2. Microcapsule Sample Preparation. For AFM measurements in the liquid, capsules needed to be immobilized within a mesh. A nylon mesh with $330 \mu \mathrm{m}$ openings (Labopolis S.L.) was glued to a Petri dish (TPP Techno Plastic Products AG, 93040) bottom using a standard two-component cyanoacrylate adhesive. Once dried, the Petri dish was carefully filled with serum-free Dulbecco's modified Eagle medium (Cultek, 12-707F), and bubbles were dislodged from the mesh by gentle pipetting. The dish was then placed and secured on a Petri dish heater (JPK instruments) assembled onto the microscope stage and incubated for $15 \mathrm{~min}$ to achieve a constant temperature of $37^{\circ} \mathrm{C}$.

A $300 \mu \mathrm{L}$-volume aliquot of the microcapsule sample was obtained under sterile conditions and gently resuspended using a specialty wide orifice tip (Sigma-Aldrich, P6800). Then, $50 \mu \mathrm{L}$ of capsule suspension was added to the Petri dish and allowed to settle in the mesh openings for $5 \mathrm{~min}$.

To perform the control AFM measurements of GO particles, the particles needed to be deposited on a surface for adequate immobilization that allowed FSBI. To this end, the GO suspension was diluted by 1:100 in distilled water and a thin layer of the suspension was pipetted on a glass coverslip. The coverslip was then immobilized on a Petri dish using a small droplet of water to leverage capillary force immobilization of the coverslip. The prepared sample was then imaged in air after calibration of the PNP-TR tip in air, using the same settings as utilized for the microcapsule FSBI. Because the particles were relatively big $(1535.33 \pm 435.31 \mathrm{~nm}),{ }^{7}$ as compared to the detection range of AFM (usually in the $100 \mathrm{~nm}$ range), it is unlikely that our stiffness measurements were affected by the substrate on which particles were deposited.

4.2.3. Force Spectroscopy-Based AFM Imaging (Quantitative Imaging). Force spectroscopy-based imaging was performed as previously described. ${ }^{22}$ Briefly, the calibrated cantilever approached toward the center of an immobilized microcapsule. Initially, quantitative imaging (QI) maps were obtained using high Z-lengths and slow speeds $\left(10 \mu \mathrm{m} \cdot \mathrm{s}^{-1}\right)$, to achieve stable measurements with a high signal/noise ratio. Then, speed was progressively increased up to $120 \mu \mathrm{m} \cdot \mathrm{s}^{-1}$ and Z-length was decreased to the $\mathrm{nm}$ scale, but programmed to at least double the image height to minimize the sample drift effects during the acquisition. To increase resolution, the piezo range was also limited to $5 \mu \mathrm{m}$. At least three capsules of each type were analyzed with at least five square images $(256 \times 256$ pixels and $\left.9 \mu \mathrm{m}^{2}\right)$ acquired per capsule. Large overview images $\left(100 \mu \mathrm{m}^{2}\right)$ were also taken to verify the homogeneity of the microcapsule surface.

4.2.4. AFM Image Processing. Images were processed with DP software (JPK Instruments, Germany). Images were first flattened using a second-order polynomial line fit to highlight smaller features from the overall curvature of the spherical sample. A low degree smoothing effect was applied to render pixel transition less evident. Finally, a gold color scale was applied to all topography images, and a blue color scale was applied to the stiffness images.

4.3. Microcapsule Constriction Assay. Microcapsule constriction was performed using a custom-made methacrylate microfluidic device, similar to a previously described system for microcapsule 
aspiration assay. ${ }^{37,38}$ The device was designed by BeOnChip S.L. (Spain) and manufactured by Aitiip Centro Tecnológico (Spain). The microdevice consists of a single $400 \mu \mathrm{m}$ tubular channel, which is reduced to $200 \mu \mathrm{m}$ (schematic of the device and dimensions are shown in Figure 5). The tube was manufactured via CNC milling of a $10 \mathrm{~mm}$-thick methacrylate plate using a two-sided milling technique. On one end, a $5 \mathrm{~mm}$ channel was milled using a $0.4 \mathrm{~mm}$ end mill. Then, a concentric $3 \mathrm{~mm}$-long channel was milled using a $14^{\prime \prime} \times 28$ UNF end mill to allow screw-on tubing connection to the device. From the other side of the plate, a concentric tubular channel was milled using a $0.2 \mathrm{~mm}$ end mill, connecting the channels on opposing sides. The same $14^{\prime \prime} \times 28$ UNF end mill was used on the second side to generate a screw-on end. Finally, a $3 \mathrm{~mm}$ end mill was used for machining the exterior of the microdevice. For the constriction assays, the microdevice was connected to a pressure controller (OB1 Microfluidic Flow Control System, Elveflow) using a 1/16"OD PTFE (Elveflow) and TYGON (Saint-Gobain, ACF00002-C) tubing systems. The microdevice was placed in an optical inverted microscope (Leica DMi8) for inspection.

4.3.1. Microcapsule Constriction Measurements. The microcapsules were diluted in DPBS. Each single microcapsule was placed in a U-shaped 96-well plate (Sarstedt) for optical microscopy inspection (Nikon-Eclipse Ti-E). Microcapsule diameters were measured with NIS Elements analysis software (Nikon) for future stiffness calculations. The constriction device was perfused with DPBS prior to the injection of each single microcapsule in the system. Pressure was slowly increased to $1000 \mathrm{~Pa}$ to position the microcapsule at the entrance of the microdevice constriction channel, avoiding mechanical damage. Once the microsphere was correctly located and blocked the flow pass, the pressure was increased at a constant rate of $100 \mathrm{~Pa} \cdot \mathrm{s}^{-1}$, causing the microcapsule to partially penetrate inside the constriction channel. This process was video-recorded, along with pressure measurements, for posterior analysis (example in Video S1). After forcing the microcapsule to go through the constricted channel, it was collected downstream in a 24 -well plate to check its integrity optically. At least 10 microcapsules per composition were tested.

4.4. Data Processing. 4.4.1. Bidimensional Statistics. Bidimensional statistical quantities (i.e., Ra, RMS, skewness, median image height, and maximum image height) were calculated using Gwyddion open-source software. ${ }^{54}$

4.4.2. Young's Moduli Extraction. Young's Moduli were extracted from nanoindentation curves acquired as pixels for the QI images. Curves were processed with DP software (JPK Instruments, Germany) and fitted to a Hertz model modified for quadratic pyramid indentors. ${ }^{41}$ The Poisson ratio was set to 0.5. Calculated values were represented in a map form, and a blue color scale was applied to all Young's moduli images. A representative force curve with the Hertz model fitting and a representative histogram of the values from a stiffness map are presented in Figure S7.

4.4.3. Correlation Analysis of Topographical and Stiffness Images. Colocalization studies of topography (gold) and stiffness (blue) maps were performed using the Van Steensel procedure in ImageJ as previously described. ${ }^{22,55,56}$ Briefly, images were converted into 16-bit binary format. For a clear representation of colocalizing features, topography images were depicted in red and topography maps in green. Pearson moment-product correlation $\mathrm{r} \rho$ was calculated and plotted against $\Delta x$. Average correlation curves + SEM were generated from at least five pairs of topography/stiffness images. Spatial Pearson moment-product correlations were compared to 0 in the central point via one-sample $t$-tests.

4.4.4. Stiffness Calculation from Constriction Data. The microcapsule stiffness was calculated as a pressure/deformation relationship $(\Delta P / \Delta \delta)$, where the deformation was identified by the penetration length percentage over the initial microcapsule diameter (Figure 5). Experimental videos were recorded simultaneously to the variation of the fluid pressure for each tested capsule (example in Video S1). The frames corresponding to discrete pressure values were exported as image files (VLC media player software). The microcapsule penetration distance in the small-diameter channel was measured using image analysis (ImageJ). The experimental results were fitted to a pressure/deformation curve, as previously reported for the mechanical characterization of capsules via aspiration assays. ${ }^{38}$

\section{ASSOCIATED CONTENT}

\section{Supporting Information}

The Supporting Information is available free of charge at https://pubs.acs.org/doi/10.1021/acsbiomaterials.0c01382.

Compression procedure in the microdevice (MP4)

General overview topographies, 3D reconstructions of detailed topographies, explanation of two-way ANOVA formulas, stiffness maps of overview and detailed maps, GO topography and stiffness analysis controls, and representative images of Young's modulus calculation process (PDF)

\section{AUTHOR INFORMATION}

\section{Corresponding Authors}

Luis Fernández - Tissue Microenvironment (TME) Lab. Aragón Institute of Engineering Research (I3A), University of Zaragoza, Zaragoza 50009, Spain; Institute for Health Research Aragón (IIS Aragón), Zaragoza 50009, Spain; Centro de Investigación Biomédica en Red en Bioingeniería, Biomateriales y Nanomedicina (CIBER-BBN), Instituto de Salud Carlos III, Madrid 28029, Spain; Email: luisf@ unizar.es

Ignacio Ochoa - Tissue Microenvironment (TME) Lab. Aragón Institute of Engineering Research (I3A), University of Zaragoza, Zaragoza 50009, Spain; Institute for Health Research Aragón (IIS Aragón), Zaragoza 50009, Spain; Centro de Investigación Biomédica en Red en Bioingeniería, Biomateriales y Nanomedicina (CIBER-BBN), Instituto de Salud Carlos III, Madrid 28029, Spain; Email: iochgar@ unizar.es

\section{Authors}

María Virumbrales-Muñoz - Department of Biomedical Engineering, Wisconsin Institutes of Medical Research, University of Wisconsin, Madison 53705, Wisconsin, United States; orcid.org/0000-0003-3660-8651

Laura Paz-Artigas - Tissue Microenvironment (TME) Lab. Aragón Institute of Engineering Research (I3A), University of Zaragoza, Zaragoza 50009, Spain; Institute for Health Research Aragón (IIS Aragón), Zaragoza 50009, Spain

Jesús Ciriza - NanoBioCel Group, Laboratory of Pharmaceutics, School of Pharmacy, University of the Basque Country (UPV/EHU), Vitoria-Gasteiz 01006, Spain; Centro de Investigación Biomédica en Red en Bioingeniería, Biomateriales y Nanomedicina (CIBER-BBN), Instituto de Salud Carlos III, Madrid 28029, Spain; 이이이.org/00000002-8666-622X

Clara Alcaine - Tissue Microenvironment (TME) Lab. Aragón Institute of Engineering Research (I3A), University of Zaragoza, Zaragoza 50009, Spain; Institute for Health Research Aragón (IIS Aragón), Zaragoza 50009, Spain; Centro de Investigación Biomédica en Red en Bioingeniería, Biomateriales y Nanomedicina (CIBER-BBN), Instituto de Salud Carlos III, Madrid 28029, Spain

Albert Espona-Noguera - NanoBioCel Group, Laboratory of Pharmaceutics, School of Pharmacy, University of the Basque Country (UPV/EHU), Vitoria-Gasteiz 01006, Spain; Centro de Investigación Biomédica en Red en Bioingeniería, Biomateriales y Nanomedicina (CIBER-BBN), Instituto de Salud Carlos III, Madrid 28029, Spain 
Manuel Doblaré - Tissue Microenvironment (TME) Lab. Aragón Institute of Engineering Research (I3A), University of Zaragoza, Zaragoza 50009, Spain; Institute for Health Research Aragón (IIS Aragón), Zaragoza 50009, Spain; Centro de Investigación Biomédica en Red en Bioingeniería, Biomateriales y Nanomedicina (CIBER-BBN), Instituto de Salud Carlos III, Madrid 28029, Spain

Laura Sáenz del Burgo - NanoBioCel Group, Laboratory of Pharmaceutics, School of Pharmacy, University of the Basque Country (UPV/EHU), Vitoria-Gasteiz 01006, Spain; Centro de Investigación Biomédica en Red en Bioingeniería, Biomateriales y Nanomedicina (CIBER-BBN), Instituto de Salud Carlos III, Madrid 28029, Spain

Kaoutar Ziani - NanoBioCel Group, Laboratory of Pharmaceutics, School of Pharmacy, University of the Basque Country (UPV/EHU), Vitoria-Gasteiz 01006, Spain; Centro de Investigación Biomédica en Red en Bioingeniería, Biomateriales y Nanomedicina (CIBER-BBN), Instituto de Salud Carlos III, Madrid 28029, Spain

Jose Luis Pedraz - NanoBioCel Group, Laboratory of Pharmaceutics, School of Pharmacy, University of the Basque Country (UPV/EHU), Vitoria-Gasteiz 01006, Spain; Centro de Investigación Biomédica en Red en Bioingeniería, Biomateriales y Nanomedicina (CIBER-BBN), Instituto de Salud Carlos III, Madrid 28029, Spain

Complete contact information is available at: https://pubs.acs.org/10.1021/acsbiomaterials.0c01382

\section{Author Contributions}

M.V.-M., J.C., L.S.d.B., J.L.P., L.F., and I.O. conceived the idea for the study. M.V.-M., L.P.-A., J.C., C.A., L.S.d.B., A.E.-N., and K.Z. performed the experiments. M.V.-M. and L.P.-A. analyzed the data. M.V.-M., J.C., K.Z., M.D., and I.O. interpreted the data. M.V.-M. and L.P.-A. wrote the manuscript and performed the statistical analyses. M.V.-M. and L.P.-A. designed the artwork; M.V.-M. created the artwork included in the article. M.V.-M., L.P.-A., J.C., K.Z., C.A., A.E.-N., L.S.d.B., J.L.P., M.D., L.F., and I.O. took part in writing the paper discussion and conclusions.

\section{Notes}

The authors declare no competing financial interest.

\section{ACKNOWLEDGMENTS}

This work has been supported by the Spanish national research program (DPI2015-65401-C3-1-R, BIO2016-79092-R and PGC2018-097257-B-C31 grants), the Aragon government (T62_20R grant) and the Basque government (consolidated groups, IT-907-16). This work has been supported by the European Union's H2020 Framework Program (H2020/20142020) and National Authorities through the Electronic Components and Systems for European Leadership Joint Undertaking (ECSEL JU) program under grant agreement Ecsel-78132-Position-II-2017-IA. Authors also wish to thank the intellectual and technical assistance from the ICTS "NANBIOSIS" Units 10 and 13 of the CIBER in Bioengineering, Biomaterials \& Nanomedicine (CIBERBBN) at the University of Basque Country UPV/EHU and the University of Zaragoza. The National Spanish Government provided M.V. studentship (FPU12/05640) and the regional Government of Aragon provided L.P. studentship. Authors gratefully acknowledge the financial support from the Centro de Investigación Biomédica en Red en Bioingenieria,
Biomateriales y Nanomedicina (CIBER-BBN). CIBER BBN is financed by the Instituto de Salud Carlos III with assistance from the European Regional Development Fund. Authors thank Dr Suzanne Ponik for her insightful comments for this manuscript.

\section{REFERENCES}

(1) Kamata, H.; Akagi, Y.; Kayasuga-Kariya, Y.; Chung, U.-i.; Sakai, T. "Nonswellable" hydrogel without mechanical hysteresis. Science 2014, 343, 873-875.

(2) Galvez-Martín, P.; Martin, J. M.; Ruiz, A. M.; Clares, B. Encapsulation in Cell Therapy: Methodologies, Materials, and Clinical Applications. Curr. Pharm. Biotechnol. 2017, 18, 365-377.

(3) Orive, G.; De Castro, M.; Ponce, S.; Hernández, R. M.; Gascón, A. R.; Bosch, M.; Alberch, J.; Pedraz, J. L. Long-term expression of erythropoietin from myoblasts immobilized in biocompatible and neovascularized microcapsules. Mol. Ther. 2005, 12, 283-289.

(4) Ciriza, J.; Saenz del Burgo, L.; Gurruchaga, H.; Borras, F. E.; Franquesa, M.; Orive, G.; Hernández, R. M.; Pedraz, J. L. Graphene oxide enhances alginate encapsulated cells viability and functionality while not affecting the foreign body response. Drug delivery 2018, 25, 1147-1160.

(5) Farina, M.; Alexander, J. F.; Thekkedath, U.; Ferrari, M.; Grattoni, A. Cell encapsulation: Overcoming barriers in cell transplantation in diabetes and beyond. Adv. Drug Delivery Rev. 2019, 139, 92-115.

(6) Medina, J. D.; Alexander, M.; Hunckler, M. D.; FernándezYagüe, M. A.; Coronel, M. M.; Smink, A. M.; Lakey, J. R.; Vos, P.; García, A. J. Functionalization of Alginate with Extracellular Matrix Peptides Enhances Viability and Function of Encapsulated Porcine Islets. Adv. Healthcare Mater. 2020, 9, 2000102.

(7) Saenz Del Burgo, L.; Ciriza, J.; Acarregui, A.; Gurruchaga, H.; Blanco, F. J.; Orive, G.; Hernández, R. M.; Pedraz, J. L. Hybrid Alginate-Protein-Coated Graphene Oxide Microcapsules Enhance the Functionality of Erythropoietin Secreting C2C12 Myoblasts. Mol. Pharm. 2017, 14, 885-898.

(8) Goenka, S.; Sant, V.; Sant, S. Graphene-based nanomaterials for drug delivery and tissue engineering. J. Controlled Release 2014, 173, $75-88$.

(9) Sanchez, V. C.; Jachak, A.; Hurt, R. H.; Kane, A. B. Biological interactions of graphene-family nanomaterials: an interdisciplinary review. Chem. Res. Toxicol. 2012, 25, 15-34.

(10) Geetha Bai, R.; Muthoosamy, K.; Manickam, S.; Hilal-Alnaqbi, A. Graphene-based 3D scaffolds in tissue engineering: fabrication, applications, and future scope in liver tissue engineering. Int. J. Nanomed. 2019, 14, 5753.

(11) Sharma, D.; Kanchi, S.; Sabela, M. I.; Bisetty, K. Insight into the biosensing of graphene oxide: Present and future prospects. Arabian J. Chem. 2016, 9, 238-261.

(12) Suk, J. W.; Piner, R. D.; An, J.; Ruoff, R. S. Mechanical properties of monolayer graphene oxide. ACS Nano 2010, 4, 65576564.

(13) Norahan, M. H.; Amroon, M.; Ghahremanzadeh, R.; Mahmoodi, M.; Baheiraei, N. Electroactive graphene oxide-incorporated collagen assisting vascularization for cardiac tissue engineering. J. Biomed. Mater. Res., Part A 2019, 107, 204-219.

(14) Ciriza, J.; Saenz del Burgo, L.; Virumbrales-Muñoz, M.; Ochoa, I.; Fernandez, L. J.; Orive, G.; Hernandez, R. M.; Pedraz, J. L. Graphene oxide increases the viability of $\mathrm{C} 2 \mathrm{C} 12$ myoblasts microencapsulated in alginate. Int. J. Pharm. 2015, 493, 260-270.

(15) Xie, H.; Yu, W.; Liu, X.; Xie, W.; Lv, G.; Wang, F.; Ma, X. Basic properties of alginate/chitosan microcapsule surfaces and their interaction with proteins. J. Controlled Release 2011, 152, No. e246.

(16) Tam, S. K.; Dusseault, J.; Polizu, S.; Ménard, M.; Hallé, J.-P.; Yahia, L. H. Physicochemical model of alginate-poly-l-lysine microcapsules defined at the micrometric/nanometric scale using ATR-FTIR, XPS, and ToF-SIMS. Biomaterials 2005, 26, 6950-6961. 
(17) Rokstad, A. M. A.; Lacík, I.; de Vos, P.; Strand, B. L. Advances in biocompatibility and physico-chemical characterization of microspheres for cell encapsulation. Adv. Drug Delivery Rev. 2014, 67-68, $111-130$.

(18) Binnig, G.; Quate, C. F.; Gerber, C. Atomic force microscope. Phys. Rev. Lett. 1986, 56, 930.

(19) Dufrêne, Y. F. Atomic force microscopy, a powerful tool in microbiology. J. Bacteriol. 2002, 184, 5205-5213.

(20) Dinarelli, S.; Girasole, M.; Longo, G. Methods for atomic force microscopy of biological and living specimens. Nanoscale Imaging; Springer, 2018, Vol. 1814; pp 529-539.

(21) Lekka, M.; Sainz-Serp, D.; Kulik, A. J.; Wandrey, C. Hydrogel microspheres: influence of chemical composition on surface morphology, local elastic properties, and bulk mechanical characteristics. Langmuir 2004, 20, 9968-9977.

(22) Virumbrales-Muñoz, M.; Santos-Vizcaino, E.; Paz, L.; GallardoMoreno, A. M.; Orive, G.; Hernandez, R. M.; Doblaré, M.; GonzalezMartin, M. L.; Fernández, L. J.; Pedraz, J. L.; Ochoa, I. Force spectroscopy-based simultaneous topographical and mechanical characterization to study polymer-to-polymer interactions in coated alginate microspheres. Sci. Rep. 2019, 9, 20112.

(23) Chui, C.-Y.; Bonilla-Brunner, A.; Seifert, J.; Contera, S.; Ye, H. Atomic force microscopy-indentation demonstrates that alginate beads are mechanically stable under cell culture conditions. J. Mech. Behav. Biomed. Mater. 2019, 93, 61-69.

(24) Iturri, J.; Toca-Herrera, J. L. Characterization of Cell Scaffolds by Atomic Force Microscopy. Polymers 2017, 9, 383.

(25) Radmacher, M.; Tillamnn, R.; Fritz, M.; Gaub, H. From molecules to cells: imaging soft samples with the atomic force microscope. Science 1992, 257, 1900-1905.

(26) Franz, C. M.; Taubenberger, A.; Puech, P.-H.; Muller, D. J. Studying integrin-mediated cell adhesion at the single-molecule level using AFM force spectroscopy. Science's STKE 2007, 2007, pl5.

(27) Hugel, T.; Seitz, M. The study of molecular interactions by AFM force spectroscopy. Macromol. Rapid Commun. 2001, 22, 9891016.

(28) Clausen-Schaumann, H.; Seitz, M.; Krautbauer, R.; Gaub, H. E. Force spectroscopy with single bio-molecules. Curr. Opin. Chem. Biol. 2000, 4, 524-530.

(29) Raab, A.; Han, W.; Badt, D.; Smith-Gill, S. J.; Lindsay, S. M.; Schindler, H.; Hinterdorfer, P. Antibody recognition imaging by force microscopy. Nat. Biotechnol. 1999, 17, 901-905.

(30) Hinterdorfer, P.; Dufrêne, Y. F. Detection and localization of single molecular recognition events using atomic force microscopy. Nat. Methods 2006, 3, 347-355.

(31) Senapati, S.; Lindsay, S. Recent progress in molecular recognition imaging using atomic force microscopy. Acc. Chem. Res. 2016, 49, 503-510.

(32) Fernandes, J. P. C.; Mareau, V. H.; Gonon, L. AFM-Raman colocalization setup: Advanced characterization technique for polymers. Int. J. Polym. Anal. Charact. 2018, 23, 113-119.

(33) Lefebvre, Y.; Leclerc, E.; Barthès-Biesel, D.; Walter, J.; Edwards-Lévy, F. Flow of artificial microcapsules in microfluidic channels: a method for determining the elastic properties of the membrane. Phys. Fluids 2008, 20, 123102.

(34) de Vos, P.; Faas, M. M.; Strand, B.; Calafiore, R. Alginate-based microcapsules for immunoisolation of pancreatic islets. Biomaterials 2006, 27, 5603-5617.

(35) Gray, A.; Egan, S.; Bakalis, S.; Zhang, Z. Determination of microcapsule physicochemical, structural, and mechanical properties. Particuology 2016, 24, 32-43.

(36) Risso, F.; Carin, M. Compression of a capsule: mechanical laws of membranes with negligible bending stiffness. Phys. Rev. E: Stat., Nonlinear, Soft Matter Phys. 2004, 69, 061601.

(37) Kleinberger, R. M.; Burke, N. A. D.; Dalnoki-Veress, K.; Stöver, H. D. H. Systematic study of alginate-based microcapsules by micropipette aspiration and confocal fluorescence microscopy. Mater. Sci. Eng., C 2013, 33, 4295-4304.
(38) Le Goff, A.; Kaoui, B.; Kurzawa, G.; Haszon, B.; Salsac, A.-V. Squeezing bio-capsules into a constriction: deformation till break-up. Soft Matter 2017, 13, 7644-7648.

(39) Cañibano-Hernández, A.; Saenz del Burgo, L.; EsponaNoguera, A.; Orive, G.; Hernández, R. M.; Ciriza, J.; Pedraz, J. L. Hyaluronic acid enhances cell survival of encapsulated insulinproducing cells in alginate-based microcapsules. Int. J. Pharm. 2019, $557,192-198$.

(40) Lin, D. C.; Dimitriadis, E. K.; Horkay, F. Robust strategies for automated AFM force curve analysis-I. Non-adhesive indentation of soft, inhomogeneous materials. J. Biomech. Eng. 2007, 129, 430-440.

(41) Hertz, H. R. Uber die Beruhrung fester elastischer Korper und Uber die Harte. Verhandlung des Vereins zur Beforderung des GewerbefleiBes 1882, 449.

(42) Silva, J. M.; Caridade, S. G.; Oliveira, N. M.; Reis, R. L.; Mano, J. F. Chitosan-alginate multilayered films with gradients of physicochemical cues. J. Mater. Chem. B 2015, 3, 4555-4568.

(43) Simpliciano, C.; Clark, L.; Asi, B.; Chu, N.; Mercado, M.; Diaz, S.; Goedert, M.; Mobed-Miremadi, M. Cross-linked alginate film pore size determination using atomic force microscopy and validation using diffusivity determinations. J. Surf. Eng. Mater. Adv. Technol. 2013, 03, 1.

(44) Raposo, M.; Ferreira, Q.; Ribeiro, P. A guide for atomic force microscopy analysis of soft-condensed matter. Mod. Res. Educ. Top. Microsc. 2007, 1, 758-769.

(45) Sobola, D.; Ţălu, Ş.; Solaymani, S.; Grmela, L. Influence of scanning rate on quality of AFM image: Study of surface statistical metrics. Microsc. Res. Tech. 2017, 80, 1328-1336.

(46) Stout, K. J. The development of methods for the characterisation of roughness in three dimensions. Commission of the European Communities: Luxembourg, 1993; Vol. 130.

(47) Orive, G.; Tam, S.; Pedraz, J.; Halle, J. Biocompatibility of alginate-poly-L-lysine microcapsules for cell therapy. Biomaterials 2006, 27, 3691-3700.

(48) Li, M.-j.; Liu, C.-m.; Xie, Y.-b.; Cao, H.-b.; Zhao, H.; Zhang, Y. The evolution of surface charge on graphene oxide during the reduction and its application in electroanalysis. Carbon 2014, 66, $302-311$

(49) Lee, K. Y.; Mooney, D. J. Alginate: properties and biomedical applications. Prog. Polym. Sci. 2012, 37, 106-126.

(50) Berger, A. J.; Renner, C. M.; Hale, I.; Yang, X.; Ponik, S. M.; Weisman, P. S.; Masters, K. S.; Kreeger, P. K. Scaffold stiffness influences breast cancer cell invasion via EGFR-linked Mena upregulation and matrix remodeling. Matrix Biol. 2020, 85-86, 8093.

(51) Wells, R. G. The role of matrix stiffness in regulating cell behavior. Hepatology 2008, 47, 1394-1400.

(52) Lim, F.; Sun, A. Microencapsulated islets as bioartificial endocrine pancreas. Science 1980, 210, 908-910.

(53) Hutter, J. L.; Bechhoefer, J. Calibration of atomic-force microscope tips. Rev. Sci. Instrum. 1993, 64, 1868-1873.

(54) Nečas, D.; Klapetek, P. Gwyddion: an open-source software for SPM data analysis. Open Phys. 2012, 10, 181-188.

(55) van Steensel, B.; van Binnendijk, E. P.; Hornsby, C. D.; van der Voort, H. T.; Krozowski, Z. S.; de Kloet, E. R.; van Driel, R. Partial colocalization of glucocorticoid and mineralocorticoid receptors in discrete compartments in nuclei of rat hippocampus neurons. J. Cell Sci. 1996, 109, 787-792.

(56) Schneider, C. A.; Rasband, W. S.; Eliceiri, K. W. NIH Image to ImageJ: 25 years of image analysis. Nat. Methods 2012, 9, 671-675. 